\title{
Congenital Goiter Associated with Cystic Hygroma Rare Cause of Stridor in New-Born - Case Report
}

\author{
Cristina Otilia Laza* and Enciu Eugenia \\ ENT/OMF Clinic, SF Apostol Andrei, Constanta, Romania, Europe
}

Submission: February 19, 2019; Published: March 05, 2019

*Corresponding author: Cristina Otilia Laza, ENT/OMF Clinic, SF Apostol Andrei, Constanta, Romania, Europe

\begin{abstract}
We present a case of a one day old neonate that appears at premature birth - 33 weeks of pregnancy with a huge cervical mass causing severe difficulties of breathing. First the child was intubated admitted in the ICU .Here my colleagues from Neonatology department struggle to sustain the vital functions with mechanical ventilator , parenteral feeding , transfusion etc. ..Diagnosis of the nature of the mass was made clinically and confirmed by ultrasonography, lab tests finally histopathology .A computer tomography of the neck, or a biopsy were not possible immediately because of prematurity , severe pulmonary complication. The surprise was to discover that the mass was a congenital goiter associated with and a right later cervical cystic hygroma -a rare lymphatic malformation. It was impossible to discover a genetic relation between the diseases or a teratogenic external cause to explain the concomitant neck lesion.

i. The purpose of the report is not only to present such a rare case, association between a giant. congenital goiter with a lymphangioma \but most important to discuss the difficulties related with the management of such a case. Example-difficult delivery., severe respiratory distress and difficulties to intubate because of deviation of the larynx.

ii. If for the diagnosis a CT scan and laboratory test are enough, is more important if the problem is diagnosed intrapartum using ultrasonography and if is an important compression on the airways a MRI will evaluate the mass for an eventual EXIT treatment.

iii. The treatment unfortunately is very difficult and dangerous because important vessel and nerves are included in the mass, plus high risk of hemorrhage, infection, plus risk of general anesthesia. Another point was our intention to discuss, what is the best attitude facing such a, problem algorithm of diagnosis and treatment.
\end{abstract}

Keywords: Infant; Stridor; Goiter; Congenital Hypothyroidism; Cervical Cystic Hygroma; Cervical Lymphangiomas; Airway Obstruction

\section{Introduction}

Congenital tumors of the cervical area are extremely uncommon in infants. Neck masses in newborns may be differentiated by their location in anterior, later cervical and posterior masses and include the following:

Cystic hygroma or lymphangioma that is the most common lymphatic malformation in children, typically presented laterocervical as a painless, trans illuminated, soft mass located superior to the clavicle; branchial cleft cysts, also laterocervical, along the anterior margin of the

\section{Sternocleidomastoid Muscle}

a. Hemangiomas, arterial malformation.

b. Flebectazia of the ijv; laterocervical.

c. External or mixt laryngocelae-also para laryngeal.

d. Thyroglossal duct cyst that may present as a midline mass.

\section{e. Teratomas. \\ f. Dermoid cyst. \\ g. Submentonier ranula.}

h. Isolated palpable cervical lymph nodes, up to $12 \mathrm{~mm}$ in diameter, are common in healthy newborns. However, lymphadenopathy may also result from congenital infection.

i. Congenital goiter (CG) is a rare cause of neonatal neck mass and may cause hyperextension of the neck.

j. Hamartomas.

k. Sarcomas, originated from a primitive soft tissue mesenchymal cell that can differentiate in many different directions-fibromatoses, fibrosarcoma's, neurofibrosarcomas, leiomyosarcomas, rhabdomyosarcomas, liposarcomas, angiosarcomas, mesothelioma. 
Differential diagnosis of fetal neck tumors is difficult only with an ultra- sonography. Prenatal diagnosis of fetal neck mass has improved the survival and morbidity of infants with giant neck masses. Prenatal ultrasonography and MRI may enhance the accuracy of antenatal diagnosis (location, extension,) and help in the selection of patients who require treatment, but fetal neck masses are uncommon and may not be apparent during the second trimester on ultrasonography. Large masses can have major fetal and perinatal effects due to the compression and distortion of surrounding cervical structures. Compression from a large lesion on the fetal esophagus and trachea can cause impaired fetal swallowing, polyhydramnios, and preterm labor in the prenatal period, and airway obstruction, hypoxia, and death after delivery. Fetal ultrasonography helps to visualize the vascularity and consistency of the mass (solid or cystic) and can determine indirect signs of esophageal or tracheal obstruction. Fetal MRI enhances sensitivity in characterizing the extent of lesion infiltration and distorted anatomy of the neck structures invasion of the large vessel and cranial nerves Prenatal MRI and ultrasonography therefore it may be critical in identifying fetal neck masses that require ex utero intrapartum treatment (EXIT) procedure.

Clinically, four common principles differentiate malign from benign:

i. Location -a superficial tumor is usually benign,

ii. Deep tumors tend to be malignant,

iii. Size- a large tumor has more chances to be malignant,

iv. Rapidly growing tumors must be suspected to be malignant,

v. Most malignant tumors are hyper vascular.

The most common treatment of choice is surgical extensive excision. Surgical resection followed by radiation is also an option for local control. Chemotherapy for sarcomas has become a more acceptable treatment modality.

\section{Case Report}

On 21 November 2016 an ENT examination was requested in the neonatology department of the hospital for a one day male newborn, born with severe stridor and respiratory distress. We found a premature - 33 weeks newborn admitted in the ICU just after the normal delivery because severe respiratory distress caused by a giant cervical mass located around the larynx.The intubation was difficult because the displacement of the larynx on the left size and put on ventilation trying to sustain the vital function On examination, patient was comatose,, not febrile,, with jaundice, pallor, body weight was $2,2 \mathrm{~kg}$, length was $39 \mathrm{~cm}$, head circumference was $30 \mathrm{~cm}$. Inspection showed an unusual hyperextension of the neck caused by a giant cervical mass measuring $14 \times 7 \times 14 \mathrm{~cm}$ extended to the neck compartments, submandibular, sub mental areas and the entire neck. The mass uncirculated the larynx with an horizontal part, and two other laterocervical bilateral parts but much bigger on the right part with displacement of the larynx and trachea to the left side, extended submandibular and sub mental areas and quite the face. On palpation, the swelling was soft, without associated inflammatory signs and tense, non-tender, mobile, non-pulsatile, non-compressible on the left laterocervical site and anterior becoming cystic fluctuant and doughy in consistency, a mass of with multiple small cysts on the right side of the neck. The remainder of the examination was unremarkable A transillumination test of the swelling on the left side with a small endoscope was positive and no bruit was audible. Examination of the mouth, nose, and throat reveals just macroglossia. Chest examination revealed decrease airway entry on left side, Broncho vascular breathing, scattered coarse crepitations, while the rest of examination was normal. No other obvious congenital anomalies were detected.

\section{History}

There was no record of thyroid disease or deafness in the family. Mother is a 28- year-old with 2 living healthy children, a spontaneous abortion on month 3 . The first were full-term, uncomplicated, pregnancies. The mother had regular antenatal visits, serologic and screening tests normal, obstetric ultrasound was done at about the 5 month of pregnancy and did not detects any abnormalities in the fetus, no other ultrasound done. No history of exposure to drugs or alcohol, use of thiourea derivatives/ antithyroid drugs (propiltiouracil, methimazole, carbimazole), iodine-rich drugs (amiodarone, antiasthma tic agents, expectorants), lithium or any other goitrogens during gestation. She had no goiter and her thyroid function was normal with negative thyroid antibodies. No other abnormalities detected during prenatal, natal and postnatal life. Mother was implicated in a car accident without victims but is starts to accuse pain, contraction on the 32 weeks, and was admitted in the hospital to prevent premature delivery but in the end, she delivers by cesarean section because at the second child delivery cesarean section was required. The Baby was delivered limp with a huge cervical mass. She was apneic, centrally cyanosed with a heart rate (HR) of 50 beats per minute. Her APGAR scores were: 0 and 6 at 1 and 5 minutes, respectively. She was resuscitated using standard resuscitation guidelines. An appropriately sized Oro-pharyngeal Airway -pipe was inserted. Initially, she was ventilated using a bag-and- mask device connected to an oxygen source. At the Neonatal Intensive Care Unit (NICU), she was intubated with an un-cuffed size $2.5 \mathrm{~mm}$ ID endotracheal tube with significant difficulties due to gross distortion of her neck anatomy. Intermittent Positive Pressure Ventilation was continued manually, and HR increased to 139 beats per minute but percutaneous oxygen saturation (SPO2) remained 60-67\%. Birth weight was $2,300 \mathrm{~g}$. 


\section{Family History}

The parents are healthy, there was no consanguinity between them. No history of family affection with same condition. There was no record of thyroid disease or deafness in the family.

\section{Laboratory Evaluation}

i. Complete blood count, reticulocyte count was in normal limits.

ii. Serum electrolytes, calcium, phosphorous, blood glucose, blood urea nitrogen, and serum creatinine: Normal.

iii. Total bilirubin: High.

iv. Tyreoglobulin dosing.

v. Dosage of anti-thyroid antibodies, anti-TPO, anti-Tg TSH.

vi. Thyroid hormones (T3, T4, FT3, FT4) (Figure 1).

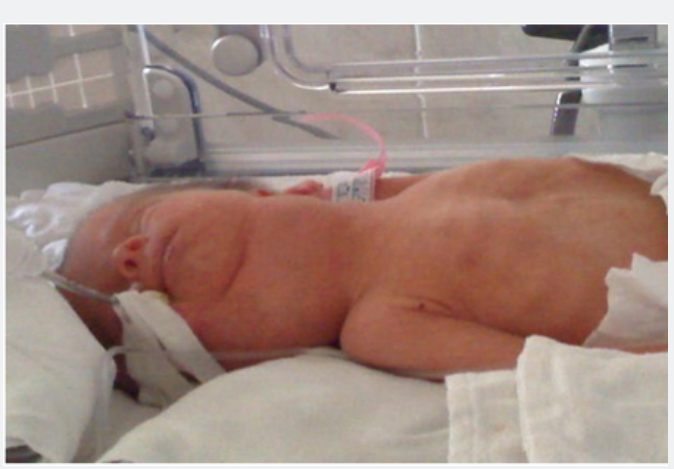

Figure 1: Newborn -with a large cervical mass intubated in the ICU -important compression on the larynx with contralateral

\section{Imagistics}

Plain X-rays of the neck was carried out and showed bilateral neck swelling without intra-thoracic extension. Neck ultrasound with Doppler reported on the anterior and left side a large thyroid gland -a goiter on the right side the gland appeared covered by a multi cystic different sized structures involving the entire right part of the neck with displacement of the airways in the left part. Doppler showed detectable vessels, the underlying neck spaces structures were compromised, large congenital bilateral neck cystic soft tissue lesion most probably lymphangioma. An ultrasonography was performed showing a marked diffuse enlargement of the thyroid gland. The findings of. hormonal replacement treatment with L-thyroxine $(25 \mu \mathrm{g} /$ day) was immediately started. Three days later, having a serum TSH of $16.6 \mu \mathrm{UI} / \mathrm{mL}$, the child was able to tolerate food. By the 12th day of hospitalization, it could be noticed the reduction of the goiter size along with muscular tonus improvement. Its course remained thereafter relatively uneventful. After 2 weeks of treatment, his free thyroxin and triiodothyronine levels normalized, and his thyroid stimulating hormone decreased to almost normal values. Therapeutic adjustment was performed the goiter decrease so much in size so detubation was possible, also feeding improved also weight, neuro and muscular development. Enhancing neck CT scan revealed a large later cervical rigid neck mass starting cranially at the level of maxillofacial regions extending caudally to the level of thoracic inlet.

The mass was composed by multiple separated cystic masses have internal enhancing septae. Deep neck structures mainly aero-digestive were pushed by the mass on the other side so the larynx and trachea were not in the midline but on direct neck examination was unable to revealed this because of the giant goiter covering the larynx and trachea. Due to the prematurity and complications - bronchopneumonia, to which extended jaundice was added, the surgical treatment was impossible so for the beginning an injection with doxycycline a scleroting agent useful for the large cysts was performed in one of the largest cysts with very good results. This procedure was repeated several times till no large cyst was visible but in the end the size of the lymphangioma was reduced to a small $3 / 2 \mathrm{~cm}$ fibrous mass with micro-cystic structure. For sure the recurrence is a rule but till than the little patients gain weight and resistance. For this reason, a long follow-up is required till surgery will be possible. For the differential diagnosis with other cervical masses CT scan, MRI and scintigraphy are essential $u$ the child was discharged on the 28th day of life with thyroid volume barely palpable, no changes in physical and neurological examination and normal neonatal hearing screening. He has continued to receive treatment with L-thyroxine and has been followed-up in the Pediatric Department of the Hospital with the support of a consultant pediatric endocrinologist. His evolution seemed to be favorable with appropriate growth and psychomotor development. The former cystic hygroma is still at the same size, periodic ultrasonography revealed only a fibrous mass without cysts. So, till now no surgery was necessary.

\section{Evaluation and Management of Neck Masses in Newborn children}

Although the diagnosis of neonatal neck mass can be made on both clinical grounds and different imaging modalities available, sometimes recognizing a mass in this region may not be easy due to the difficulty of examining the neck of neonates

\section{Clinical Presentation}

Clinical presentation of a large tumor in early neonatal life is a. Polyhidramnios.

b. Respiratory distress, stridor caused by the compression and displacement to of the masses on the larynx and trachea and possible mediastinal involvement.

c. Dysphagia-compression on the pharynx or esophagus

d. Infections. 
e. Heart failure.

f. Spontaneous bleeding or rupture of the mass.

g. Death especially when there are coexisting malformations.

\section{Clinical Examination}

a) Inspection

b) Palpation

c) Auscultation

Can offer information about the size, region, consistency, solid or cystic mass or polycystic, tender or no, inflammation, adherence to the deep structures, relation with the airways and with the large vessels of the neck. Such information is vital-ex.

a. In the midline are only tyreoglossal tract cyst or teratoma or thyroid related masses

b. Laterocervical.

c. Unicystic mass is a branchial cyst.

d. Multicystic mass represents a lymphangioma.

e. Pulsation means arterial malformation.

f. Compresible-ectazia of the IJV, laryngoele.

The clinical findings presenting with goiter vary from asymptomatic to enlarged thyroid volume causing stridor, cyanosis and respiratory distress by airway obstruction that can be a serious emergency.

\section{Imaging}

Trans-illumination: Ancient method to diagnose a cystic hygroma.

Ultrasonography: A noninvasive, easy to use, movable and cheap method, very good to diagnose a $\mathrm{CH}$ is a multi-cystic lesion with internal septation and no blood flow on color, also a goiter or the lymph nodes.

Ultrasonography: Ideal to evaluate the salivary glands also.

Doppler Ultrasonography: For the large vessels of the neck.

X-ray: Neck front/prophile -laryngocelae is visible cyst depressible filled with air.

X-ray lung: Attention of a possible mediastinum extension.

CT Scan: Most important investigation capable to evaluate the nature of the mass, extension, cystic component, or solid nature, contrast substance intake, true extension, invasion or displacement of vital structures like cranial nerves, large vessels, airways - larynx, trachea, esophagus; pharynx, in salivary glands, extension in the mediastinum.

MRI ScanL: Is more useful in diagnosis of a soft tumor.
Angio CT: Is important to diagnose a vascular malformation like haemangioma or flebectazia of Internal Jugular Vein, also the relation of the mass with the large vessels of the neck

Biopsy/excision biopsy with histology was unachieved in our case, mature teratomas most frequently show adult type tissue derided from the embryonic layers especially skin and adnexa, bronchial mucosa and gland, as well as gastrointestinal mucosa.

Prenatal Diagnosis of a cystic hygroma or a teratoma has been described using ultrasonography. Teratomas are typically seen as large asymmetric, well defined, one sided neck masses often associated with polyhydriamnos. Cystic hygroma is often identified as a multilocular fluid-filled cavity visible as increased nuchal translucency greater than 99th percentile for gestational age in the first trimester.

The differential diagnosis of cervical masses in neonates includes

i. Lymphatic malformation.

ii. Congenital goiter.

iii. Thyro-glossal duct.

iv. Brachial cleft cysts.

v. Arteriovenous malformation.

vi. Lipoma.

vii. Laryngocele.

viii. Neuroblastoma.

ix. Cervical teratomas, neoplasms consisting of ectodermal, mesodermal, and endodermal germ with various degrees of differentiation. Cervical teratomas are uncommon in neonates, accounting for approximately 3-5\% of all teratomas.

\section{Congenital Goiter}

Congenital goiter is a rare cause of neonatal neck mass. Most cases of congenital hypothyroidism $(\mathrm{CH})$ are not hereditary and results from thyroid dysgenesis, maternal ingestion of antithyroid drugs, goiterogens, transplacental passage of maternal antibodies and rare causes like activating mutations of the TSH receptor, activating mutations of the G- protein $\alpha$ subunit (McCune Albright syndrome), tumors. Even in the hereditary forms of goiter and thyroid dysfunction that often accompanies it may not be evident at birth [1]. The prevalence of the $\mathrm{CH}$ based on nationwide programs for neonatal screening is $1 / 4000$ infants worldwide. Twice as many girls as boys are affected. Dysharmonogenesis represents $10-15 \%$ of all the causes of $\mathrm{CH}$ and most neonates would exhibit a relatively large goiter. Majority of case reports on neonatal goiter in literature were based on recording of fetal goiter by antenatal scans which is dependent on radiologist's expertise. Furthermore, there is lack of ultrasonography machines and qualified 
manpower to carry out routine antenatal scans in resource poor nations. Congenital hypothyroidism is a syndrome caused by insufficient supply of tissues with thyroid hormones at birth. It is characterized by neurological immaturity, mix edematous infiltrate, hypometabolism. Thyroid hormones are vial for myelinization and correct neural connections, the critical period being in the first few months of life. HC is the most common childhood endocrine disease. Worldwide prevalence of 1 / 3,000 to $1 / 5,000$ in the newborn, higher in geographic areas deficient in iodine in water and in the soil, called gusogenic, or endemic. Severe mental retardation such as spasticity, dysarthria, mutism, autism is the most serious effect of untreated hypothyroidism, as well as altered bone growth and maturation [2-5].

There are two forms of endemic cretinism:

a) Neurological-mental retard mental form, spasticity, ataxia, deafness-mutitis, - development of normal-deficiency of iodine in fetal life.

b) Mixed-form-nanism, dismorphism, mental retardation without other troubles. Neurological.

\section{Classification of Etiology}

\section{Hormonogenesis disorders:}

i. Inability to capture iodine,

ii. Congenital disorders of the TSH receptor,

iii. Inability to degrade thyroglobulin,

iv. Sdr. Pendred-deafness association.

Congenital disorders of thyroid hormone receptors in tissues.

Very rarely is a thyroid disorder or a treatment applied to the mother during pregnancy:

i. Lack or excess of iodine,

ii. Radioactive treatment,

iii. Excess antithyroid therapy propylthiouracil, methimazole sulfonamide-lithium- phenylbutazone -pas or iodides cross the placenta and block the fetal thyroid gland.

iv. Goiterogens

v. Soybeans, cabbage

Autoimmune mother disease with anti-thyroid antibody transfer through placenta.

Hypopituitarism - TSH-TRH deficiency.

Gland infiltration diseases - Hystiocitosis X, cystinosis.

\section{Clinical Signs}

\section{Birth}

a. Overload of pregnancy. b. High birth weight.

c. Persistence of neonatal jaundice.

d. Hoarseness, dyspnea, cyanosis, hypothermia, bradycardia

The newborn with congenital hypothyroidism at 4-8 weeks has.
a. Macroglossia - tongue increases in volume.
b. Large abdomen umbilical hernia.
c. Dry, pale skin, cold.

d. Normal weight increases but not in length.

e. Delayed bone growth, appearance of disproportionate nonsense, large head, short neck, short limbs to the body, small nose, large mouth, lips. After 2 years, the mental development retardation is obvious: the speech appears late, the child is apathetic, sleepy, with disturbance of attention, memory. There may be idiotic, deafness, deafness. The child does not increase enough in height, has walking disorders, dental disorders, later puberty disorders [6-10].

\section{Diagnosis}

a) Tyreoglobulin dosing.

b) Dosage of anti-thyroid antibodies, anti-TPO, anti-Tg TSH.

c) Thyroid hormones (T3, T4, FT3, FT4).

d) Ultrasonography.

e) Thyroid scintigraphy may be helpful).

f) Radiological examinations reveal bone age retardation.

g) Genetic counseling is also recommended.

h) CT scan of the neck and thorax-mediastinum extension.

Occasionally this disorder may be identified prenatally with the chance of prenatal treatment of such cases by injecting T4 into the amniotic fluid. Thus, it is important to highlight the role of strict ultrasound monitoring during future pregnancies in order to detect fetal goiter. A Diagnosis of neonatal dysharmonogenitic goiter with hypothyroidism once made. thyroxin treatment is started on $15 \mathrm{mcg} / \mathrm{kg} /$ day and by 15 th day decreased in the size of the goiter with regular passage of motions and good cry and activity is noticed. The main goals of the treatment are to assure normal growth and development and psychometric outcome similar to genetic potential, by restoring the serum $\mathrm{T} 4$ concentration as rapidly as possible to a normal range followed by continued clinical and biochemical euthyroidism. Compliance to treatment plan, periodic follow-up care and adjustment of therapy are essential pieces for a good outcome. In many countries, including Romania, hypothyroidism is screened by 
TSH or T4 dosing by the dry spot on filter paper. For prophylaxis, the authorities have the obligation to supplement the iodine intake of the endemic inhabitants, by the iodization of the kitchen salt, of the marketed food [11-15].

\section{Cystic Hygroma}

Cystic Hygroma is a rare benign cystic lymphatic malformation also known cystic lymphangioma, Hygroma is a term with a Greek origin meaning "tumor containing water". First described by Wernher in 1843, cystic hygroma is a benign tumor multicystic tumor with a very rare incidence $-1 / 16.0001$ new-born. Even they can appear anywhere, in head and neck area are almost $3 / 4$ of all, most frequent on the left later cervical area, most frequent on the posterior triangle of the neck and on the left side, extension in the mediastinum is possible. In almost all the patients at birth they are already present, sometimes giant with respiratory distress.

\section{Pathogenesis}

Cystic hygromas are congenital malformations of the lymphatic system; developmental lymphangiomas derived from the primitive embryonic jugular venolymphatic sacs

Congenital lymphangiomas arise from a combination of causes:

a. Failure of lymphatics to connect with the venous system.

b. Abnormal budding of lymphatic tissue.

c. Sequestered lymphatic tissue with their embryonic growth potential intact.

This is the reason for a fast development, plus the lymphatic rests can penetrate adjacent structures or dissect along fascial planes. In the end become canalized., retain their secretions because the connection with venous outflow tract is absent. That's how cystic component is explained.. The tumor has indiscrete margins and is composed of dilated cystic spaces lined by endothelial cells. The lack of encapsulation results in difficult surgical removal and incomplete resection, which in turn results in frequent recurrences. Cystic hygromas typically grow gradually, with infrequent spontaneous regression

In congenital hygromas most of the patients presents

i. -karyotypic abnormalities and

ii. -are more common in persons with Turner, Down, Klinefelter syndrome, and trisomy 18 and 13.

iii. -also are associated with, non-chromosomal disorders, including Noonan syndrome, Fryns syndrome, multiple pterygium syndrome, and achondroplasia.

iv. Intrauterine alcohol exposure is associated with hygroma v. Acquired lymphangiomas arise after a neck trauma (including surgery), inflammation, or obstruction of a lymphatic drainage pathway.

\section{Signs and Symptoms}

a. The initial manifestations of cystic hygroma are intrauterine and include,

b. Polyhydramnios,

c. Fetal hydrops,

d. Hyperechogenic lung fields, and

e. Inverted diaphragms.

The polyhydramnios is secondary to compression of the upper esophagus. Alveoli distal to the upper airway obstruction become dilated. The microcystic form tends to predominate in the oral cavity and oropharynx-as clusters of clear, black, or red vesicles on the buccal mucosa or tongue. Macrocystic lymphomas tend to develop below the mylohyoid muscle and can involve both the anterior and posterior triangles of the neck. The cysts are typically large and thick walled, and the overlying skin can take on a bluish hue or may appear normal. A sudden increase in size secondary to infection or intralesional bleeding is possible. Spontaneous decompression or shrinkage is uncommon. Potentially life-threatening airway compromise with noisy breathing (stridor) inspiratory bradipneea and cyanosis is possible in large lymphangiomas with laryngeal, tracheal displacement. Feeding difficulties, as well as failure to thrive, when the lesion affects structures of the upper digestive tract. pharynx, esophagus [16-18].

\section{Clinical Examination}

On inspection, palpation, the typical hygroma appear as a soft, painless, compressible (doughy) mass that usually trans illuminates with in case of stridor is important to evaluate, laryngeal tracheal deviation or other evidence of impending airway obstruction. Inspection of the tongue, oral cavity, hypopharynx, and larynx is important because any involvement of these structures may lead to airway obstruction. The examination is completed with a fiberoptic endoscopy with airway evaluation also pharyngo-esophageal examination performed by a trained ENT specialist.

\section{Complications}

Prenatal diagnosis affords the opportunity to plan a surgical approach and to evaluate and secure an airway.

a. Airway obstruction, most common life-threatening complication.

b. Hemorrhage.

c. Infection may lead to rapid increase in size, with subsequent airway and esophageal compression. 
d. The mass is often too large to allow vaginal delivery without encountering airway complications and consequent anoxic brain injury to the infant.

e. Deformation of surrounding bony structures or teeth (if an LM is left untreated).

\section{Diagnostic Considerations}

\section{Laboratory Studies}

i. Prenatal fluorescent in-situ hybridization (FISH) can be used to evaluate for lymphatic malformation.

ii. Prenatal chromosomal analysis for chromosomes 13, 18, 21, $\mathrm{X}$, and $\mathrm{Y}$.

\section{Imaging Studies}

Prenatal diagnosis affords the opportunity to plan a surgical approach and to evaluate and secure an airway compression.

\section{Plain Radiography}

In any large mass of the head and neck, anteroposterior and lateral neck radiographs can be helpful in delineating possible airway compromise, but information's offered are limited compared with the risk of irradiation.

\section{Ultrasonography}

This is the least invasive study. It is very useful to reveal the cystic nature of the mas and the relationship with the surrounding structures. but is limited in evaluation of the extension in the mediastinum and in the retropharyngeal space. Ultrasonography is very useful to detect hygroma in utero and is important in detection airway compression.

\section{Magnetic Resonance Imaging}

MRI it provides the best soft tissue detail and can delineate the relationship of the lesion to underlying structures. Contrast can be used to differentiate hemangiomas from lymphangiomas. On MRI, hygroma appear as hyperintense on T2-weighted images and hypointense on T1-weighted images.

\section{Computed Tomography}

CT is faster than MRI and may be more readily available. It carries the risk of radiation exposure, and detail is lost if the LM is surrounded by tissue of similar attenuation. Contrast helps to enhance cyst wall visualization and the relationship to surrounding blood vessels. 3D Reconstruction on CT is useful in respiratory distress caused by a large lymphatic malformation. Moreover, it is desirable to diagnose CT prenatally in order to plan delivery and postnatal care in a designated center to improve outcome. Nonetheless, early prenatal diagnosis was not attained in our patients, apparently due to sub-optimal antenatal care and non-availability of ultrasound scanning machines in the referring rural facilities. Pre-operative computerized tomography is important to evaluate extension of the masses, their association with neurovascular structures, so we can prevent iatrogenic damage during surgical excision and enhance prognosis (Figure 2).

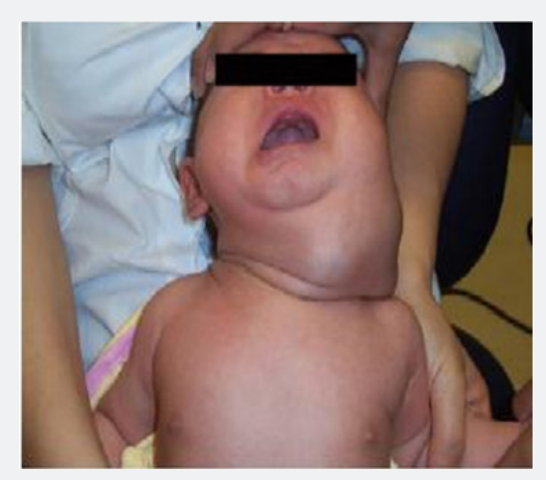

Figure 2: Infant with a large late rocervical mass.

\section{Ultrasound-Guided Biopsy}

\section{Histologic Findings}

Cystic hygromas are composed of large irregular sinuses with a single layer of flattened epithelial lining and fibrous adventitial coats. The thickness of the vessel wall varies, with both striated and smooth muscle components. Although are multicystic, a unilocular cyst is found in approximately $10 \%$ of cases. Cysts can range from $1 \mathrm{~mm}$ to several centimeters in size and are filled with clear- to straw-colored fluid, which is eosinophilic and protein-rich. Individual cysts may be isolated or may freely communicate. The surrounding stroma is fibrous or fatty and may contain lymphoid aggregates, smooth muscle, or other local tissues. LMs may contain multiple subtypes (e.g., capillary and cavernous) and, in these instances, are categorized according to the predominant subtype. In addition, lesions with a hemangiomatous component are considered hemangiolymphangiomas. Hemorrhage into the cyst is common and can be secondary to trauma or spontaneous bleeding. Capillary hygroma contain capillary-sized lymphatic channels that involve the epidermis. Cavernous type infiltrate surrounding structures and are dilated lymphatic channels. Macrocystic types are cystic masses lined by a single layer of endothelium with a connective tissue stroma.

\section{Classification}

a) In 1877, the first system was proposed by Wegener.

b) In 1982, Mulliken and Glowacki.

c) Hemangiomas.

d) Vascular malformations including lymphangiomas

e) The World Health Organization (WHO) recognizes three types of lymphangiomas:

f) Capillary.

g) Cavernous, and 


\section{h) Cystic.}

Giguere proposed a classification based on the size of the cystic component,

a. Macrocystic - Cystic spaces $\geq 2 \mathrm{~cm}$.

b. Microcystic - Spaces $<2 \mathrm{~cm}$.

c. Mixed lesions.

d. De Serres et al proposed the following system for staging of lymphangiomas.

e. Stage I - Unilateral infrahyoid (17\% complication rate).

f. Stage II - Unilateral suprahyoid (41\% complication rate).

g. Stage III - Unilateral and both infrahyoid and suprahyoid (67\% complication rate).

h. Stage IV - Bilateral suprahyoid (80\% complication rate).

i. $\quad$ Stage V - Bilateral infrahyoid and suprahyoid (100\% complication rate (Figure 3).

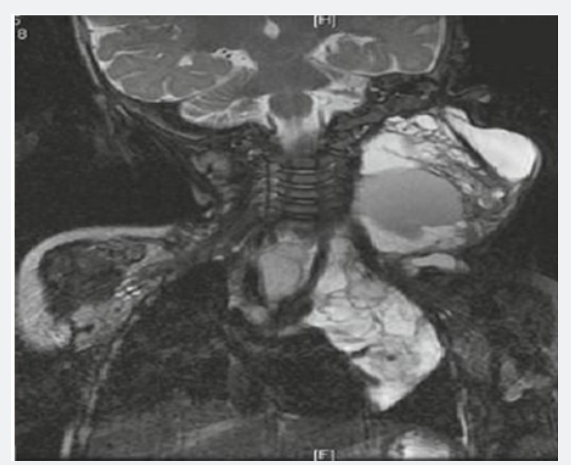

Figure 3: MRI frontal View -Cystic Hygroma Cervical and Thoracic.

\section{Treatment and Prognosis}

\section{Medical Care}

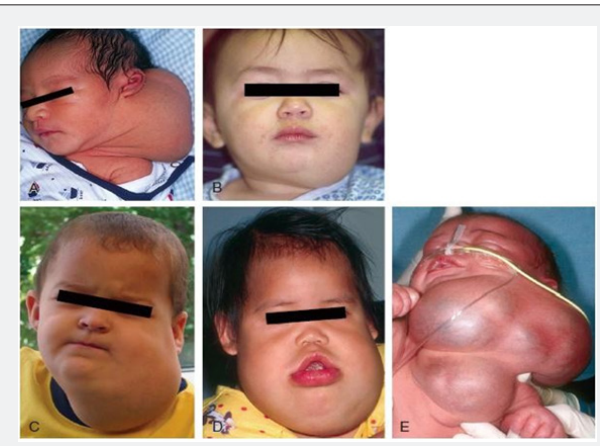

Figure 4: Head and neck lymphatic malformation stages. A, Stage 1, unilateral infrahyoid. B, Stage 2, unilateral suprahyoid. C, Stage 3, unilateral suprahyoid and infrahyoid. D, Stage 4, bilateral suprahyoid. E, Stage 5, bilateral suprahyoid and infrahyoid.
Although some authors have reported watchful waiting for lymphatic malformations this should be considered only in patients who are asymptomatic. Medical treatment of LMs consists of the administration of sclerosing agents, such as OK432 (an inactive strain of group A Streptococcus pyogenes), bleomycin, pure ethanol, bleomycin, sodium tetradecyl sulfate, and doxycycline. An infected LM should be treated with intravenous antibiotics, and definitive surgery should be performed once the infection has resolved (Figure 4).

\section{Surgical Care}

The mainstay of treatment of LMs is surgical excision. The surgical team should attempt to remove the LM completely or, failing that, to remove as much as possible, sparing all vital structures like cranial nerves, major vascular structures, or hypopharynx, parotid gland, or trachea. Complete excision is possible only $40 \%$ of cases. The procedure is rare possible at the time of diagnosis -causes include premature infants who are small in size and neurovascular structures are involved but small and difficult to identify (VII CN). If no airway obstruction is present, Surgery can be delayed until 2 years or more if airway obstruction is absent and the parotid gland is involved. In case of airway obstruction, we can avoid an emergency tracheostomy, using aspiration with an 18-gauge or 20-gauge needle of large cyst but recurrence is a rule and complications like hemorrhage, or infection may appear. Although traditional aspiration is often followed by recurrence, hemorrhage, or infection.

Radiofrequency ablation (RFA) can be used in case on intraoral extension, especially microcystic lesions.

Magnetic resonance-controlled laser-induced interstitial thermotherapy is a newer therapy proposed for treatment of LM.

\section{Ex utero Intrapartum Treatment}

Ex utero intrapartum treatment (EXIT) is defined as a partial delivery of the fetus through a lower transverse hysterotomy by ultrasound guidance, performed while maintaining maternalfetal blood flow. EXIT is required in large obstructing airway mass, like hygroma, teratoma or rhabdomyosarcoma). If such a mass is visible on prenatal ultrasonography, MRI should be performed to identify the nature of the mass, extensions. A multispecialty team, including a high-risk obstetrician, pediatric otolaryngologist, pediatric surgeon, and neonatologist, is required in case of ex-utero intrapartum treatment (EXIT). A planned cesarean delivery is performed, and intubation or tracheostomy is used to establish an airway. Extracorporal membrane oxygenation (ECMO) should also be available. Excision of the LM is delayed until the child is stable. Intrauterine cyst aspiration to facilitate vaginal delivery is possible .

\section{Complications}

i. Damage of one or more cranial nerves-VII, X, XI, XII.

ii. Chylous fistula. 
iii. Chylothorax.

iv. Damage of a large vessel Hemorrhage.

v. Infection.

vi. Perforation of the pharynx, oesophagus or trachea.

vii. Recurrence - Most recurrences develop within the first year, but some have arisen as long as 10 years after excision.

viii. Multiple operations may be necessary to remove complex lesions, particularly those with extension into the mediastinum. Recurrence rates depend on the complexity of the lesion and degree of excision. Incompletely excised lesions may recur in $50 \%$ to $100 \%$ of cases.

\section{Conclusion}

Congenital tumors are extremely uncommon in infants, particularly in the head and neck region. The most common fetal neck mass is cystic higroma; cervical teratoma is the most common the fetal neck tumor. but association with a goiter is very rare. Differential diagnosis of fetal neck tumors is difficult to be detected by ultrasonography. lymphangioma/higroma, teratoma, sarcoma, haemangioma, neuroblastoma, and goiter should be included in the differential diagnosis of fetal neck masses. Prenatal diagnosis of fetal neck mass has improved the survival and morbidity of infants with giant neck masses Prenatal ultrasonography and MRI may enhance the accuracy of antenatal diagnosis (location, extension, and intracranial spread) and help in the selection of patients who require treatment, but fetal neck masses are uncommon and may not be apparent during the second trimester ultrasonography. In this case report, we present a heterogeneous mass arising from the right lateral neck with radiological, histological, and immune-histochemical findings. Cystic hygroma is a rare lymphatic malformation in children, and it should be considered in the differential diagnosis of congenital neck masses. Giant ones are life-threatening conditions leading to airway obstruction, with high risk of recurrence.

MRI examination is of great benefit for correct evaluation, extent of the lesion and thus selection of the correct and success of the treatment. In addition, current trend in the management of cystic hygroma includes Ex. utero Intra-partum Treatment (EXIT) procedure to secure the newborn airway at delivery while still on placental support and early surgical extirpation of the tumor. The tumor should be completely removed in order to prevent recurrence or malignant transformation. Similarly, a complete surgical excision of $\mathrm{CH}$ is the treatment of choice. Other management modalities include cauterization, marsupialization and sclerotherapy with bleomycin, triamcinolone or fibrin sealant. Also, radiofrequency ablation may be used in persistent or unresectable $\mathrm{CH}$. $\mathrm{CH}$ reoccurs in about $20 \%$ of cases after treatment. Surgeons should ensure total resection of the mass without any remnant is performed and that the unity of vital tissues is conserved during surgery. Non-surgical treatment modalities for cystic hygroma especially intralesional sclerosing agent's administration like bleomycin, doxicicline, triamcinolone or fibrin sealant should be considered as the primary form of treatment for lymphangiomas especially in children with severe general problems like in our case due to good results and minimal complication rate.

In conclusion, large cystic tumors-like hygromas or teratomas also large goiters are rare but potentially lifethreatening neonatal emergencies due to the associated severe airway obstruction. Prenatal diagnosis via optimal prenatal ultrasonography will ensure well planned deliveries and favorable outcome of these rare congenital disorders in resource- limited settings. Goiters in newborn infants are not seen frequently but all pediatricians who deal with neonates should be in a position to recognize the syndrome, understand its cause and prognosis and to advise therapy. Fast diagnosis of congenital hypothyroidism and hormonal treatment are able not only to reduce the goiter but to permit a good development of the child .A long follow-up, adjustment of the doses is important. A giant cervical mass in infants is a serious problem requiring a multidisciplinary team including -neonatologist, pediatrician, ENT surgeon skilled in cervical surgery and airways desobstruction, anesthesiologist, vascular surgeon, pediatric surgeon, specialist in ultrasonography, radiology, imagistic, endocrinologist.

\section{References}

1. American Academy of Pediatrics, Rose SR; Section on Endocrinology and Committee on Genetics (2006) American Thyroid Association, Brown RS; Public Health Committee, Lawson Wilkins Pediatric Endocrine Society, Foley T, Kaplowitz PB, Kaye CI, Sundararajan S, Varma SK. Update of newborn screening and therapy for congenital hypothyroidism. Pediatrics 117(6): 2290-303.

2. Crawford JD (1956) Goiters in childhood. Pediatrics 17(3): 437-441.

3. Everett LA, Glaser B, Beck JC, Idol JR, Buchs A, et al. (1997) Pendred syndrome is caused by mutations in a putative sulphate transporter gene (PDS). Nature Genet 17(3): 411-422.

4. Felner EI (2002) A newborn with a goiter and thyroid dyshormonogenesis. J Maternal-Fetal \& Neonatal Med 12(3): 207-208.

5. Gow L, Gulati R, Khan A, Mihaimeed F (2011) Adult-onset cystic hygroma: a case report and review of management. Grand Rounds 11: 5-11.

6. Guruprasad Y, Chauhan DS (2012) Cervical Cystic Hygroma. J Maxillofac Oral Surg 11(3): 333-336.

7. La Franchi S, Kirkland JL, Ross D, Hoppin A, Mulder J (2010) Congenital and acquired goiter in children. Up to Date, Waltham, Massachusetts.

8. La Franchi S (2010) Treatment and prognosis of congenital hypothyroidism. In: Rose BD, editors. UpToDate. Waltham, Massachusetts.

9. Medeiros-Neto GA, Stanbury JB (1994) Inherited disorders of the thyroid system. CRC Press. Boca Raton, Florida.

10. Muir A, Daneman D, Daneman A, Ehrlich R (1988) Thyroid scanning, ultrasound and serum thyroglobulin in determining the origin of congenital hypothyroidism. Am J Dis Child 142(2): 214-216. 
11. Karakas O, Karakas E, Boyaci FN, Murat Yildizhana, Songul Demir, et al. (2013) Cervicomediastinal Giant Cystic Hygroma. A Case Report. J Clin Med Res 5(1): 61-63.

12. Kim DW (2014) OK-432 sclerotherapy of lymphatic malformation of the head and neck: factors related to outcome. Pediatric Radiology 44(7): 857-857.

13. Sandrucci MA, Nicolin V, Forabosco A, Narducci P, Bareggi R, et al. (2002) Morphological observations and morphometric analysis in three human fetuses with bilateral cervical cystic hygroma. Ital J Anat Embryol 107(1): 57-70.

14. Sanhal CY, Mendilcioglu I, Ozekinci M, Yakut S, Merdun Z, et al. (2014) Prenatal management, pregnancy and pediatric outcomes in fetuses with septated cystic hygroma. Braz J Med Biol Res 47(9): 799 -803.
15. Sannoh S, Quezada E, Merer DM, Moscatello A, Golombek SG (2009) Case Report: Cystic hygroma and potential airway obstruction in a newborn: a case report and review of the literature. Cases Journal 2: 48.

16. Southgate WM, Pittard WB (2001) Classification and physical examination of the newborn infant. In: Klaus MH, Fanaroff AA, editors. Care of the high-risk neonate. 5th ed. Philadelphia: WB Saunders pp. 100 .

17. Sherman BE, Kendall K (2001) A unique case of rapid onset of a large cystic hygroma in the adult. Am J Otolaryngol 22(3): 206-210.

18. Suwanrath Kengpol C, Suntharasa T, Patrapinyokul S, Chanvitan P (2003) Prenatal diagnosis of a huge cystic hygroma colli. Ultrasound Obstet Gynecol 22(3): 323-324.

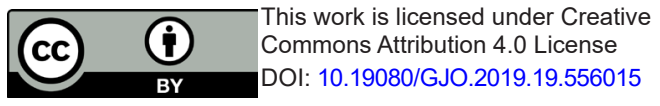

\begin{tabular}{l} 
Your next submission with Juniper Publishers \\
will reach you the below assets \\
- Quality Editorial service \\
- Swift Peer Review \\
- Reprints availability \\
- E-prints Service \\
- Manuscript Podcast for convenient understanding \\
- Global attainment for your research \\
- Manuscript accessibility in different formats \\
( Pdf, E-pub, Full Text, Audio) \\
- Unceasing customer service \\
Track the below URL for one-step submission \\
https://juniperpublishers.com/online-submission.php \\
\hline
\end{tabular}

